\title{
Resonance of terahertz phonons in an acoustic nanocavity
}

\author{
S. Sandeep, ${ }^{1}$ S. L. Heywood, ${ }^{2}$ R. P. Campion, ${ }^{2}$ A. J. Kent, ${ }^{2}$ and R. N. Kini ${ }^{1, *}$ \\ ${ }^{1}$ School of Physics, Indian Institute of Science Education and Research Thiruvananthapuram (IISER-TVM), Vithura, Maruthamala \\ Post Office, Thiruvananthapuram, Kerala, 695551, India \\ ${ }^{2}$ School of Physics and Astronomy, University of Nottingham, University Park, Nottingham NG7 2RD, United Kingdom
}

(Received 14 September 2018; revised manuscript received 27 November 2018; published 21 December 2018)

\begin{abstract}
We show the resonant behavior of $\sim 0.5-\mathrm{THz}$ longitudinal acoustic (LA) phonons in an acoustic nanocavity of thickness $\sim 28 \mathrm{~nm}$ sandwiched between two GaAs/AlAs superlattices (SLs). One of the SLs, upon excitation with an ultrafast optical pulse, acts as a source of coherent LA phonons. These phonons, generated outside the cavity, resonantly drive the cavity mode. The phonon dynamics can be modeled using a driven-damped harmonic oscillator. The confinement and enhancement of phonons in the acoustic nanocavity opens up possibilities in the field of nanophononics.
\end{abstract}

DOI: 10.1103/PhysRevB.98.235303

\section{INTRODUCTION}

In several musical instruments, vibrations in a column of air are used to produce musical notes. These vibrations are loudest (resonant) for certain frequencies which depend mainly on the length and geometry of the tube containing the air column. The relative strength and the resonant frequencies of vibrations differ between instruments, giving rise to a unique tone for each instrument. Similar resonance phenomena can be observed for terahertz $(\mathrm{THz})$ "sound" using an acoustic nanocavity. Analogous to an optical microcavity which confines the photon field and modifies the light-matter interaction in the cavity, an acoustic nanocavity confines and amplifies acoustic phonons in the $\mathrm{THz}$ range. The confined phonons form a standing wave in the cavity. The decay of the confined mode depends on the quality factor $Q$ of the cavity, which is defined as the frequency-to-bandwidth ratio of the cavity mode. It has been shown that high- $Q \mathrm{THz}$ phonon cavities can be fabricated and the $Q$ is mainly limited by submonolayer thickness fluctuations [1]. Direct laser excitation of a cavity phonon mode $(\sim 0.55 \mathrm{THz})$ in a $\lambda / 2$ GaInAs cavity sandwiched between two phonon Bragg mirrors has been demonstrated [2]. Compared to the superlattice (SL) phonon modes, the cavity modes have a long lifetime $(\sim 350 \mathrm{ps})$ due to the high $Q$ of the cavity. By using a double resonant cavity, where the same $\lambda / 2$ cavity confines both photons as well as acoustic phonons, strong enhancement in the interaction of the photons and phonons of the same wavelength has been reported [3]. The confined acoustic phonon cavity mode could be probed using Raman scattering since embedding the phonon cavity in an optical microcavity enhances the optical field in the cavity [4]. In most of these cases, acoustic phonons have been generated in the cavity by direct ultrafast laser excitation of the cavity [2,3,5]. Using thin aluminum layers as broadband sources of sub-THz acoustic phonons, evidence for resonant transmission of cavity modes $(\sim 100 \mathrm{GHz})$ through

\footnotetext{
*rajeevkini@iisertvm.ac.in
}

an acoustic nanocavity has been provided [6]. In the present work we seek the possibility of driving the cavity mode using an external $\mathrm{THz}$ acoustic phonon source. We show the resonant behavior of $\sim 0.474 \mathrm{THz}$ longitudinal acoustic (LA) phonons in an acoustic nanocavity, generated by ultrafast excitation of a SL located outside the cavity. This is a step towards the experimental realization of the "phoniton" and other interesting phenomena related to the interaction of phonons with a two-level electron system (TLS) in an acoustic cavity [7]. For realizing a cavity phoniton, which involves the short-range interaction of electrons and cavity phonons, (i) a high- $Q$ acoustic phonon cavity, (ii) a TLS driven directly by the cavity phonon mode, and (iii) strong coupling between the TLS and the cavity phonon mode are required [7]. A double quantum well can act as a TLS since the degeneracy of the ground state of its individual quantum wells can be lifted by applying a bias across the quantum wells [8,9]. It has been shown earlier that in quantum wells, the electrons couple strongly to LA phonons due to deformation-related variation of the effective mass mechanism [10]. Hence if an electrically biased double quantum well (TLS) is enclosed in a high- $Q$ phonon cavity, it will be possible to strongly couple and drive the TLS using the cavity phonon mode. Here, we show that it is possible to excite the cavity mode, using an external phonon source, which can potentially be used to drive the TLS and observe the cavity phoniton phenomena.

\section{THE DEVICE AND THE EXPERIMENT}

Figure 1(a) schematically shows the device structure. Periodic semiconductor layers or SLs based on GaAs and AlAs are the building blocks of the device. An acoustic cavity of length $5 \lambda / 2$ is sandwiched between two SL structures which act as Bragg mirrors for acoustic phonons of wavelength $\lambda$. The semiconductor layers as shown in Fig. 1(a) were grown by molecular beam epitaxy (MBE) on $\sim 0.4$ mm-thick (001) semi-insulating GaAs substrate. The top SL consisted of 11 periods, each period comprising 7.1-nm GaAs and 2.8-nm AlAs, $n$ doped with $\mathrm{Si}$ to a concentration of $2 \times 10^{18} \mathrm{~cm}^{-3}$. 
(a)

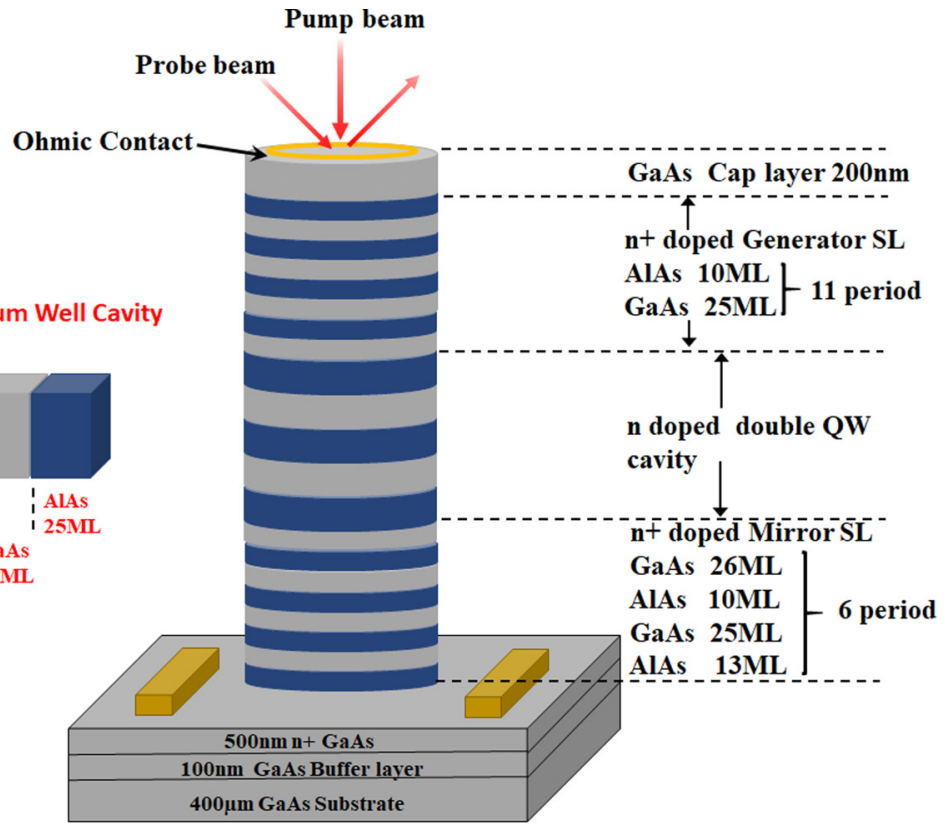

(b)

5 $\lambda / 2$ Double Quantum Well Cavity
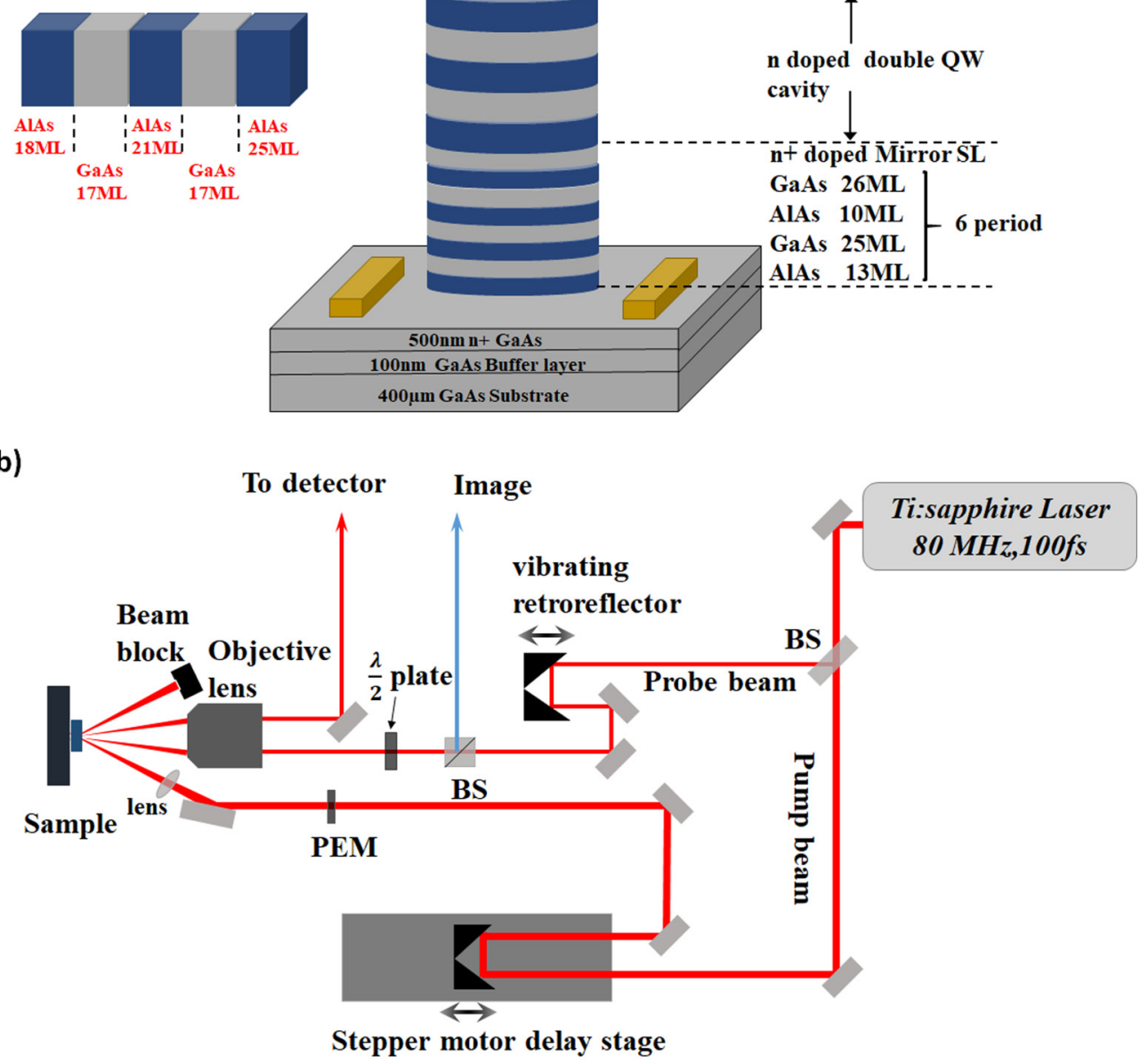

Pump beam: P-Polarized

Probe beam: S-Polarized

FIG. 1. (a) The phonon cavity structure. (b) Schematic of the pump-probe experimental setup.

The bottom SL consisted of six periods, with each period comprising 7.3-nm GaAs, 2.8-nm AlAs, 7.1-nm GaAs, and 3.7-nm AlAs, uniformly $n$ doped with Si to a concentration of $2 \times 10^{18} \mathrm{~cm}^{-3}$. The $5 \lambda / 2$ cavity consisted of a uniformly $n$-doped $\left(5 \times 10^{16} \mathrm{~cm}^{-3}\right) \mathrm{GaAs}$ double quantum well (well width $4.8 \mathrm{~nm}$ ) with AlAs barriers, with a total length of $28 \mathrm{~nm}$. Using conventional photolithographic techniques, the structure was processed into $200-400-\mu \mathrm{m}$-diameter devices.

The phonon dynamics were studied using a standard degenerate $(736 \mathrm{~nm})$ pump-probe technique in the reflection geometry, schematically shown in Fig. 1(b), using $\sim 100$-fs pulses at a repetition rate of $80 \mathrm{MHz}$, from a mode-locked Ti:sapphire laser [11]. The top SL generated coherent phonons when excited by a femtosecond laser (pump) pulse with a photon energy near or above the lowest electron-hole resonance of the generation SL, which is $1.68 \mathrm{eV}$ in this case
[12-14]. The generation and detection of coherent phonons in SLs have been experimentally demonstrated previously [15]. The pump beam was chopped at a frequency of $50 \mathrm{KHz}$ using a photoelastic modulator and then focused to a spot diameter of $\sim 25 \mu \mathrm{m}$ using a lens of focal length $50 \mathrm{~mm}$. The probe was focused to a size of $\sim 6 \mu \mathrm{m}$ on the sample using a reflective microscope objective. The probe monitored the reflectivity changes in the GaAs cap layer as well as the top few layers of the generator SL. The laser mean power was $25 \mathrm{~mW}$ for the pump pulse and $1 \mathrm{~mW}$ for the probe pulse. In the probe path a vibrating retroreflector was introduced which allowed scanning of the delay time between the pump and the probe at $\sim 2.5 \mathrm{~Hz}$. A stepper motor delay line in the pump path allowed measurements to be taken for long delay ranges. The changes in the reflected intensity of the probe, $\Delta R(t)$, were monitored using a photodiode and the photodiode signal was averaged 


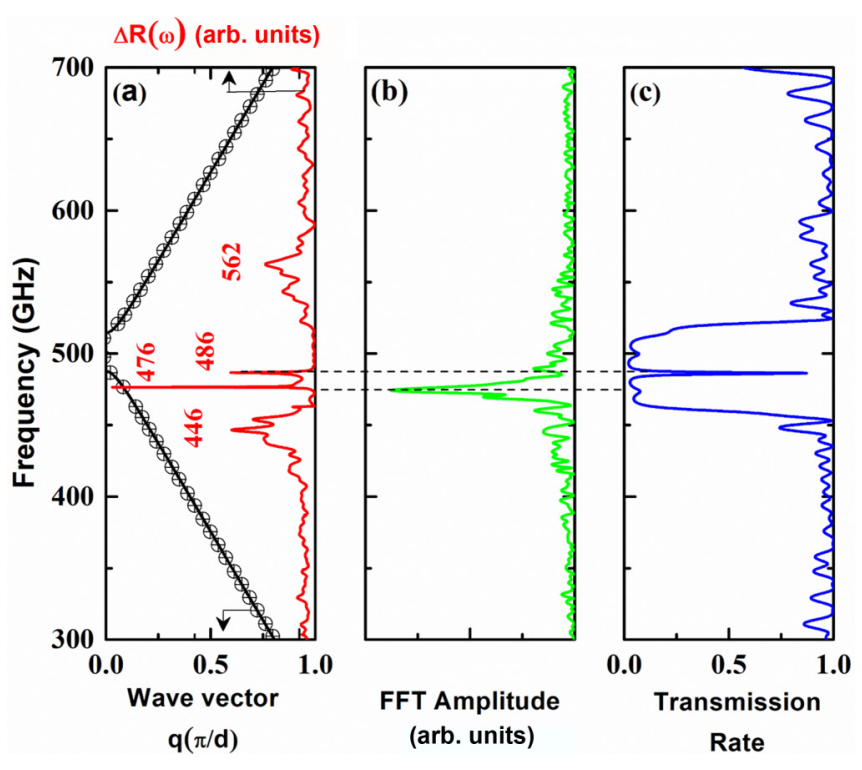

FIG. 2. (a) Black line: Folded phonon dispersion calculated for the top SL. Circle: Normal modes of the complete structure. Red line: calculated spectral response $\Delta R(\omega)$ of the structure. The two peaks seen at 476 and $486 \mathrm{GHz}$ are the generation mode of the structure; the other two peaks at 446 and $562 \mathrm{GHz}$ are the detection response of the structure. (b) Fourier transform amplitude of the reflectivity signal $(0-450 \mathrm{ps})$. (c) Acoustic transmission rate for the entire structure. Dip at $486 \mathrm{GHz}$ represents the cavity mode.

using a lock-in amplifier which was referenced to the pump chopper frequency. The output of the lock-in amplifier was fed to an averaging oscilloscope which was synchronized with the vibrating retroreflector in the probe path. The vibrating retroreflector arrangement allowed recording of the data with very high sensitivity in 45-ps fragments, and by fast Fourier transforming (FFT) these fragments the oscillation frequency in different time windows was obtained. The device was mounted on the cold finger of an ultralow vibration closed cycle refrigerator to do measurements at low temperature $(\sim 14 \mathrm{~K})$.

The top SL also serves to generate $\sim 0.474 \mathrm{THz}$ LA phonons upon ultrafast photoexcitation [14]. The spectral response of the structure in a pump-probe experiment $\Delta R(\omega)$ can be calculated considering (i) the generation of coherent acoustic phonons and (ii) the detection spectral response of the acoustic phonons in the structure $[15,16]$. Figure 2(a) shows the calculated spectral response, $\Delta R(\omega)$, of the structure along with the LA phonon dispersion of the top SL calculated using the Rytov model [17]. The two peaks seen around the $q=0$ edge of the dispersion, at 476 and $486 \mathrm{GHz}$, are the generation [forward scattering (FS)] modes of the complete structure. When we calculated the generation spectral response for the top SL alone, we observed a single FS mode at $476 \mathrm{GHz}$. For the complete structure an additional peak is found at 486 $\mathrm{GHz}$, which is a normal mode. We attribute this extra mode to the finiteness of the structure [16]. For a finite structure, only certain modes obeying the boundary conditions called normal modes are allowed, which are also indicated in Fig. 2(a). These discrete modes of an acoustic field are obtained by imposing a stress-free boundary condition at the free end of the

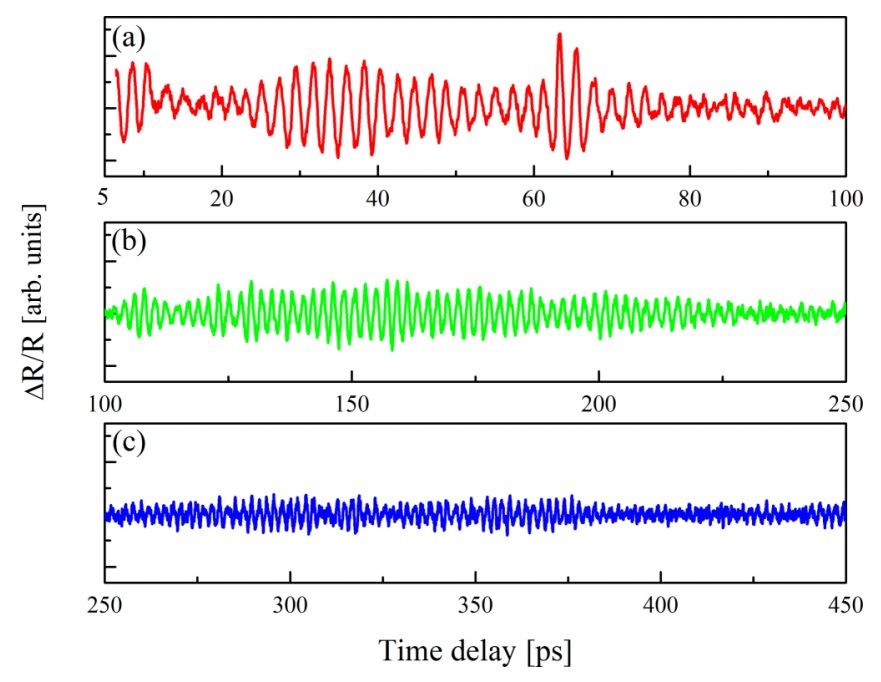

FIG. 3. (a-c) Oscillatory component of the reflectivity signal as a function of the delay between the pump and the probe in three different time windows.

structure and a zero acoustic displacement boundary condition at the opposite end of the structure. In Fig. 2(a), the peaks observed at 446 and $562 \mathrm{GHz}$ are the backscattering (BS) modes. The acoustic reflection coefficient of the structure for LA phonons, calculated using the transfer matrix method, is shown in Fig. 2(c) $[18,19]$. The dip at $\sim 486 \mathrm{GHz}$ represents the acoustic cavity mode [4]. The structure is designed in such a way that the cavity mode matches one of the generation modes of the top SL.

\section{RESULTS AND DISCUSSION}

Figures 3(a)-3(c) show the oscillatory component of the transient reflectivity signal in the temporal range 0-450 ps. The electronic decay contribution and Brillouin oscillations have been filtered out in order to clearly see the phonon oscillation in the reflectivity signal. We have observed the maximum acoustic phonon oscillation when the excitation wavelength was $\sim 736 \mathrm{~nm}$ which is $\sim 80 \mathrm{meV}$ above the electron-hole resonance energy of the top SL. The frequency of the dominant oscillations is observed at $474 \mathrm{GHz}$ [Fig. 2(b)] which corresponds to the FS generation mode of the top SL and matches very well with the calculated generation mode.

In order to further understand the dynamics of oscillations, the amplitude of the oscillations was calculated by FFT of the $\Delta R(t)$ fragments in smaller time windows $(\sim 20 \mathrm{ps})$, which is shown in Figs. 4(a) and 4(e); here the time axis indicates the center of the windows. The initial $\sim 100$ ps shows a beating of two modes with a frequency difference of $\sim 31.5 \mathrm{GHz}$. As shown in Fig. 4(b), the FFT of the oscillations in the 5-100 ps range shows two frequency components at 474 and $444 \mathrm{GHz}$ which are the FS and BS modes of the top SL, respectively. Hence we can understand the beating pattern in the FFT amplitude in the initial $\sim 100 \mathrm{ps}$ range to be due to FS and BS modes of the structure $[12,14,20]$. For $t>100 \mathrm{ps}$, the detection mode at $444 \mathrm{GHz}$ is found to vanish rapidly due to its short lifetime when compared to the generation mode. 

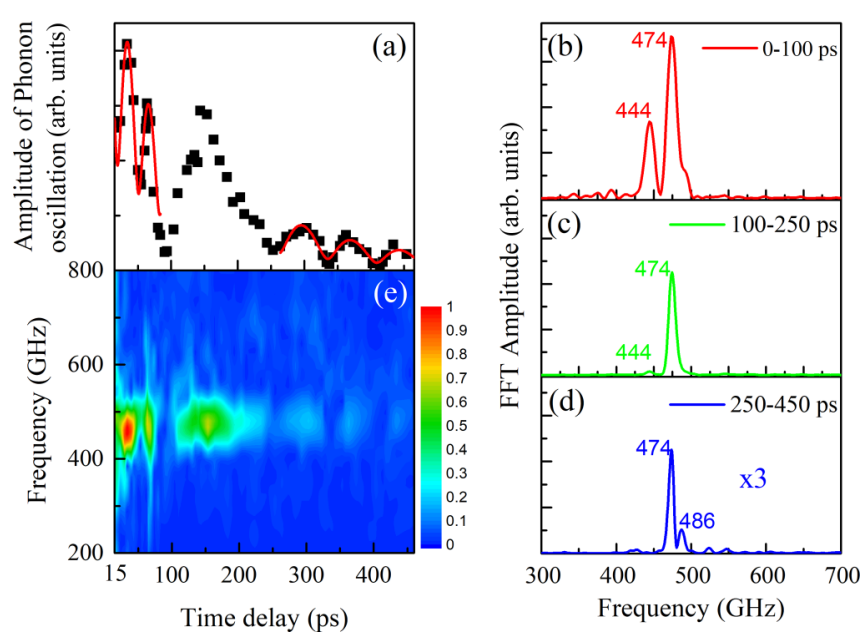

FIG. 4. (a) Fourier transform amplitude of the transient reflectivity signal in 20-ps intervals. The time axis indicates the center of the intervals. The solid line is a guide to the eye and represents two beating cosines. (b-d) Fourier spectra of the transient reflectivity signal in the three time intervals indicated. (e) Color map of the Fourier transform's amplitude in 20-ps intervals as a function of frequency and time delay.

In the $\sim 100-250$ ps time range we see a nonmonotonic behavior, with an increase in amplitude initially and then an exponential decrease. The time taken for a round trip (from the top of the structure to the cavity and back) by LA phonons, generated by ultrafast pulsed excitation of the top SL, is about $\sim 108$ ps, which matches well with the start of the buildup of the oscillation amplitude. The FFT of the $\Delta R(t)$ fragment in the $\sim 100-250$ ps time range shows a prominent peak at $\sim 474 \mathrm{GHz}$ which corresponds to the generation mode. We believe that the top SL acts as a driving force for the cavity and $\mathrm{THz}$ oscillations build up in the cavity. The oscillation amplitude reaches a maximum at around $150 \mathrm{ps}$ and then decays afterwards. In the later time scale (250-450 ps), the amplitude of oscillation shows a beating of two modes with a frequency difference of around 13.6 GHz. Fourier transform of the reflectivity signal between 250 and 450 ps [Fig. 4(d)] shows peaks around 474 and $486 \mathrm{GHz}$. As discussed earlier these frequencies correspond to the generation mode of the top SL and the cavity mode, respectively. The beating of these two modes contributes to the beating pattern for $t>250 \mathrm{ps}$.

To explain the dynamics of the $\Delta R(t)$ signal, we have developed an analytical model where we considered the cavity as a driven-damped harmonic oscillator with a resonant frequency, $\omega_{0}(486 \mathrm{GHz})$. The cavity is driven by the generation normal modes $\left(\omega_{1}=474 \mathrm{GHz}, \omega_{2}=486 \mathrm{GHz}\right)$. The experimentally generated spectrum [Fig. 4(b)] is broad compared to the calculated spectrum, and contains frequency components at both 474 and $486 \mathrm{GHz}$. Hence it is reasonable to assume that the cavity is driven by both modes. The analytical model can be expressed as

$$
\frac{d^{2} x}{d t^{2}}+2 \Gamma \omega_{0} \frac{d x}{d t}+\omega_{0}^{2} x=e^{-\gamma t}\left[\cos \left(\omega_{1} t\right)+B \cos \left(\omega_{2} t\right)\right]
$$
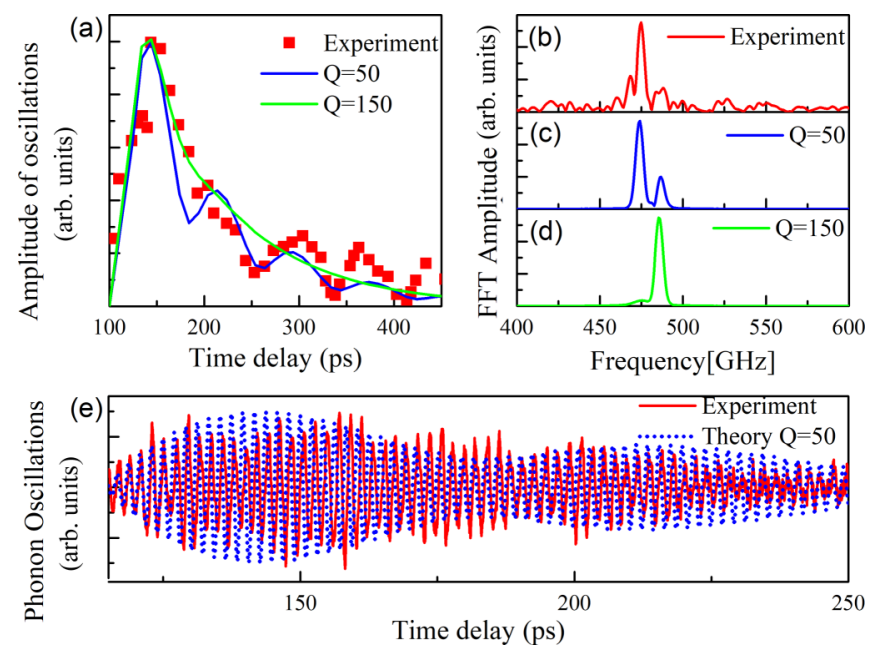

FIG. 5. (a) Amplitude of phonon oscillation in the cavity calculated for two different $Q$ s shown along with the experimentally observed phonon amplitudes. (b) Fourier transform of the experimental reflectivity signal. (c,d) Fourier spectra of the calculated phonon oscillation for two $Q$ values. (e) Oscillatory component of the resolved reflectivity signal in the time window 110-250 ps shown along with the calculated amplitude of oscillations in the cavity for $Q=50$.

where $x$ is the amplitude of oscillation, $\Gamma$ is related to the $Q$ of the cavity $(Q=1 / 2 \Gamma)$, and $\gamma$ is the damping constant of the driving force which depends on the lifetime of the coherent phonons. $B$ is the ratio of the amplitude of the two modes. By numerically solving the above equation we can obtain the amplitude of the oscillations in the cavity and match this to the experimental result by using as fitting parameters the $Q$ of the cavity and the decay constant of the driving force $\gamma$. We found that a good match between the experimental and calculated phonon amplitudes is obtained for $Q \precsim 150$. In Fig. 5(a) the amplitude of phonon oscillation in the cavity, obtained by FFT of the calculated phonon oscillations in 20 ps time intervals, is shown along with the experimentally observed phonon amplitudes. One can see that a very good agreement of the oscillation frequency and the buildup and decay dynamics is obtained for $Q \sim 50$ and $\gamma=0.0067 \mathrm{ps}^{-1}$. This indicates that the top SL modes acts as the driving force for the cavity, and the externally generated $474 \mathrm{GHz}$ phonon mode forms a standing wave in the cavity, which leads to the buildup of the $474 \mathrm{GHz}$ mode in the cavity. For $Q \sim 150$ and $\gamma=0.05 \mathrm{ps}^{-1}$, it was observed that the cavity buildup matches well with experimentally observed one [Fig. 5(a)], but, as shown in Fig. 5(d), the numerically obtained frequency of these oscillations is at $486 \mathrm{GHz}$ (cavity mode), whereas, experimentally, we observed a buildup in the phonon amplitude with dominant frequency at $474 \mathrm{GHz}$ (driving mode) which is followed by a beating of 474 and $486 \mathrm{GHz}$ modes beyond $t \sim 250 \mathrm{ps}$. The $Q$ value $(\sim 50)$ obtained from the fitting is significantly lower than the cavity $Q$ value of 340 for the $486 \mathrm{GHz}$ cavity mode determined from the acoustic reflection coefficient of the structure [Fig. 2(c)]. This may be due to submonolayer thickness fluctuations in the SL layers [1]. In order to estimate the effect of thickness fluctuations on the $Q$ of the cavity, we introduced random disorder of \pm 1 
atomic monolayer to the layer thicknesses and calculated the transmission spectra. Averaging 150-1000 spectra gave a $Q$ value $\sim 45$, which matches well with our observation.

\section{CONCLUSIONS}

In conclusion, we have designed and fabricated an acoustic phonon cavity using GaAs/AlAs SL Bragg mirrors. The top SL mirror was excited using an ultrafast laser pulse which generated $474 \mathrm{GHz}$ LA phonons. These modes generated outside the cavity were used to force the cavity into oscillation. We observed a buildup of the driving mode in the cavity. This is similar to the case of the resonance tube experiment performed in undergraduate laboratories in which the resonance phenomenon is observed when the length of the air column is a multiple of the quarter-wavelength of sound produced by a tuning fork. Instead, here we use a 28-nm-long "sound" column, along with a source of $\mathrm{THz}$ sound to do an analogous experiment at the nanoscale.

Even though the $Q$ of the cavity used in the current experiment is low, this experiment paves the way for the observation of more interesting physical phenomena like the phonon analog of cavity-quantum electrodynamics. For example, by using a very high- $Q$ phonon cavity $\left(Q \sim 10^{4}-10^{6}\right)$ and by introducing a TLS whose energy is in resonance with the cavity mode, it may be possible to observe vacuum Rabi splitting of the phonon mode [7]. The GaAs/AlAs double quantum well can act as a TLS since the energy level splitting $\Delta$ between the ground states of the quantum wells can be changed and brought in resonance with the cavity mode by applying a bias across the quantum wells $[8,9]$. To observe Rabi splitting of the phonon mode, strong coupling between the TLS and the cavity phonon mode is required. In quantum heterostructures, the interaction between electrons and LA phonons is strongly enhanced compared to bulk semiconductors, due to deformation-related variation of the effective mass mechanism [10]. Hence when $\Delta$ exactly matches the cavity phonon energy, the electron should undergo intersubband electronic transitions between the ground state of the two quantum wells with the absorption and emission of cavity phonons, leading to the observation of Rabi splitting of the cavity phonon mode.

\section{ACKNOWLEDGMENTS}

The authors acknowledge funding support from the Department of Science and Technology (DST), India, and the UK-India Education and Research Initiative (UKIERI) during the initial part of this work. R.N.K. acknowledges funding support from the Kerala State Council for Science, Technology and Environment (KSCSTE), Kerala, India. S.S. acknowledges useful discussions with A. Fainstein.
[1] G. Rozas, M. F. Pascual Winter, B. Jusserand, A. Fainstein, B. Perrin, E. Semenova, and A. Lemaître, Phys. Rev. Lett. 102, 015502 (2009).

[2] M. F. Pascual Winter, G. Rozas, A. Fainstein, B. Jusserand, B. Perrin, A. Huynh, P. O. Vaccaro, and S. Saravanan, Phys. Rev. Lett. 98, 265501 (2007).

[3] A. Fainstein, N. D. Lanzillotti-Kimura, B. Jusserand, and B. Perrin, Phys. Rev. Lett. 110, 037403 (2013).

[4] M. Trigo, A. Bruchhausen, A. Fainstein, B. Jusserand, and V. Thierry-Mieg, Phys. Rev. Lett. 89, 227402 (2002).

[5] N. D. Lanzillotti-Kimura, A. Fainstein, A. Huynh, B. Perrin, B. Jusserand, A. Miard, and A. Lemaitre, Phys. Rev. Lett. 99, 217405 (2007).

[6] A. Huynh, N. D. Lanzillotti-Kimura, B. Jusserand, B. Perrin, A. Fainstein, M. F. Pascual-Winter, E. Peronne, and A. Lemaître, Phys. Rev. Lett. 97, 115502 (2006).

[7] Ö. O. Soykal, R. Ruskov, and C. Tahan, Phys. Rev. Lett. 107, 235502 (2011).

[8] A. J. Kent, R. N. Kini, N. M. Stanton, M. Henini, B. A. Glavin, V. A. Kochelap, and T. L. Linnik, Phys. Rev. Lett. 96, 215504 (2006).

[9] B. A. Glavin, V. A. Kochelap, and T. L. Linnik, Appl. Phys. Lett. 74, 3525 (1999).
[10] V. I. Pipa, N. Z. Vagidov, V. V. Mitin, and M. Stroscio, Phys. Rev. B 64, 235322 (2001).

[11] R. P. Beardsley, A. V. Akimov, M. Henini, and A. J. Kent, Phys. Rev. Lett. 104, 085501 (2010).

[12] D. C. Heinecke, O. Kliebisch, J. Flock, A. Bruchhausen, K. Köhler, and T. Dekorsy, Phys. Rev. B 87, 075307 (2013).

[13] A. Huynh, B. Perrin, and A. Lemaître, Ultrasonics 56, 66 (2015).

[14] A. Bartels, T. Dekorsy, H. Kurz, and K. Köhler, Phys. Rev. Lett. 82, 1044 (1999).

[15] M. Trigo, T. A. Eckhause, J. K. Wahlstrand, R. Merlin, M. Reason, and R. S. Goldman, Appl. Phys. Lett. 91, 023115 (2007).

[16] M. F. Pascual-Winter, A. Fainstein, B. Jusserand, B. Perrin, and A. Lemaître, Phys. Rev. B 85, 235443 (2012).

[17] S. M. Rytov, Sov. Phys. Acoust. 2, 68 (1956).

[18] S. Tamura, D. C. Hurley, and J. P. Wolfe, Phys. Rev. B 38, 1427 (1988).

[19] S. Mizuno and S. I. Tamura, Phys. Rev. B 45, 734 (1992).

[20] F. Sun, Q. Wu, Y. L. Wu, H. Zhao, C. J. Yi, Y. C. Tian, H. W. Liu, Y. G. Shi, H. Ding, X. Dai, P. Richard, and J. Zhao, Phys. Rev. B 95, 235108 (2017). 